\section{Associação entre a violência psicológica e o transtorno de estresse pós-traumático em adolescentes de uma coorte}

\section{Association between psychological violence and posttraumatic stress disorder in a cohort of Brazilian adolescents}

\section{Asociación entre violencia psicológica y trastorno de estrés postraumático en adolescentes de una cohorte}

Livia Goreth Galvão Serejo Álvares 1

Maria Teresa Seabra Soares de Britto e Alves 1

Alcione Miranda dos Santos 1

Bruno Luciano Carneiro Alves de Oliveira 2

Deysianne Costa das Chagas 1

doi: 10.1590/0102-311X00286020

\section{Resumo}

Este trabalho analisou a influência da ocorrência de violência psicológica no desenvolvimento de transtorno de estresse pós-traumático (TEPT) em adolescentes. Tratou-se de um estudo transversal aninhado a uma coorte, cujo segundo segmento foi realizado em 2016. Aplicou-se um questionário em 2.486 adolescentes, por meio do qual se abordou aspectos individuais, familiares, sociais e da vivência de violência psicológica. Utilizou-se o escore de propensão para se criar o inverso da probabilidade de seleção (IPS). Dessa forma, atribuiu-se uma probabilidade para cada adolescente, sendo 1/IPS aqueles que compõem o grupo dos expostos e 1/(1-IPS) aqueles do grupo dos não expostos. Essa condição tornou os dois grupos mais homogêneos e comparáveis entre si. A associação entre a ocorrência de violência psicológica e de TEPT foi estimada pela odds ratio (OR), enquanto o intervalo de 95\% de confiança (IC95\%) foi estimado por meio da regressão logística binária bruta e ajustada, ponderada pelo IPS. Verificou-se que 30,3\% relataram ter sofrido violência severa. A prevalência do TEPT foi de 4,8\% entre os expostos contra $1,5 \%$ entre os não expostos à violência psicológica. Observou-se uma associação da violência severa com o TEPT nas duas análises realizadas. Porém, a magnitude no modelo estruturado pelo escore de propensão $(O R=1,97 ;$ IC95\%: 1,08-3,56) indicou um ajuste da medida de associação da análise bruta (OR = 3,40; IC95\%: 2,035,69). Nesse sentido, este estudo contribui para a escassa literatura sobre a exposição à violência psicológica e a sua associação com o desenvolvimento de TEPT, confirmando o impacto negativo dessa forma de abuso na saúde mental do individuo.

Adolescente; Violência; Transtorno de Estresse Pós-Traumático
Correspondência

L. G. G. S. Álvares

Universidade Federal do Maranhão.

Av. dos Portugueses 1966, São Luís, MA 65080-805, Brasil.

livia.goreth@ufma.br

1 Universidade Federal do Maranhão, São Luís, Brasil. 2 Universidade Federal do Maranhão - Campus Pinheiro, Pinheiro, Brasil. 


\section{Introdução}

Durante a adolescência, o indivíduo se depara com diversas situações conflitantes que resultam, na maioria das vezes, em alterações comportamentais que podem camuflar uma situação de violência vivenciada 1,2,3. Dentre essas situações, a violência interpessoal, seja ela sofrida ou presenciada, funciona como um importante preditor para o desenvolvimento de psicopatologias, que geram fortes prejuízos sociais e profissionais $1,3,4,5$.

Ainda são escassos os estudos que analisam a violência psicológica de maneira isolada, isto é, como um evento estressor associado ao desenvolvimento de transtorno de estresse pós-traumático (TEPT). Muitas vezes, tal forma de violência é associada às demais, o que não só a negligencia, mas também lhe confere poder detentor de maiores efeitos danosos às vítimas 1,5,6. Isto pode ser explicado pelo quão recente é a atenção voltada para este tipo de abuso ${ }^{5}$. As situações de violência psicológica destroem a autoimagem do adolescente e provocam traumas que afetam o seu psiquismo. Assim, o indivíduo assume atitudes e emoções que o levam a uma incapacidade de interagir socialmente com as condições próprias da sua idade, uma vez que situações como estas podem torná-lo passivo ou mesmo agressivo $3,5,7$.

O TEPT é um transtorno psiquiátrico e debilitante que se encontra entre os transtornos mentais mais frequentes, em decorrência da exposição às mais variadas formas de violência. Geralmente, afeta indivíduos vulneráveis de qualquer faixa etária, pois todos estão sujeitos a um evento traumático. Contudo, requer-se uma atenção especial para indivíduos na adolescência, período em que a individualidade é desenvolvida e os danos repercutem não só a curto e médio prazos, como também na vida adulta $1,6,7,8$.

A prevalência de TEPT varia amplamente entre estudos e sofre influência da diversidade do contexto socioeconômico e cultural de cada população estudada 1,5,9,10. Muito do que se sabe sobre a prevalência de TEPT em adolescentes advém de pesquisas realizadas em vítimas expostas à perda de um ente querido, à desastres naturais e à violência comunitária, física e sexual. As taxas em estudos internacionais variam entre 4,7\% e 7,6\%, em que os extremos são mais frequentes em vítimas de violência sexual 1,3,4,5,11,12.

Lewis et al. 13, em um estudo de coorte, mostraram que 31,1\% dos adolescentes foram expostos a diferentes traumas e 7,8\% desenvolveram TEPT aos 18 anos. O risco de desenvolver TEPT foi maior após exposição à violência interpessoal. Esta categoria de trauma esteve associada a quase metade dos casos de TEPT.

Sullivan et al. 14, avaliando adolescentes vítimas de diferentes tipos de violência interpessoal, encontraram que a violência psicológica se apresentou como o único preditor significativo de gravidade do TEPT, cujo predomínio foi do sexo feminino. Os autores afirmaram que a violência psicológica pode ser mais prejudicial do que as outras formas de violência devido ao seu caráter silencioso e à ausência de evidências físicas. Entre as limitações dessa análise, os autores citaram a inclusão de algumas formas de abuso, como o bullying, perpetrado por familiares e colegas.

No Brasil, a prevalência de TEPT variou entre 6,5\% e 9,5\% em estudos em que foram utilizadas amostras com crianças e adolescentes vítimas de violência comunitária 15. Em uma análise mais recente, Avanci et al. 16 encontraram uma prevalência de 7,8\% em adolescentes de baixa renda vítimas de violência física e psicológica. O transtorno de estresse pós-traumático é um dos transtornos mais comuns associados à exposição à violência. No entanto, há informações limitadas sobre o seu desenvolvimento, tanto de maneira isolada quanto associado à ocorrência de violência psicológica 3,4,5.

Analisar a violência psicológica como risco para o desenvolvimento de TEPT em adolescentes se torna relevante a partir do momento em que se fornece informações de um grupo populacional em que a vulnerabilidade é muito presente. Estes dados, além de úteis, tornam-se necessários à prestação de cuidados adequados tanto para a prevenção primária quanto secundária do TEPT. Portanto, em vista dos riscos apresentados ao desenvolvimento do adolescente, é fundamental o apoio às políticas públicas de enfrentamento de estados de vulnerabilidade. Ciente disto, este estudo objetivou analisar a influência das ocorrências de violência psicológica, em sua forma severa, associada ao desenvolvimento de TEPT. 


\section{Materiais e métodos}

Trata-se de estudo transversal aninhado a um estudo de coorte com indivíduos nascidos na cidade de São Luís, Maranhão, Brasil. Essa coorte está incluída no consórcio de coortes RPS, um consórcio de coortes brasileiras de nascimento de Ribeirão Preto, Pelotas e São Luís - desenvolvido pela Universidade Federal do Maranhão (UFMA), pela Faculdade de Medicina de Ribeirão Preto da Universidade de São Paulo (USP) e pela Universidade Federal de Pelotas (UFPel). Neste artigo, utilizou-se dados de São Luís, que possui, de acordo com o último censo realizado pelo Instituto Brasileiro de Geografia e Estatísticas (IBGE) 17, 1.014.837 pessoas e renda mensal em média de 3,1 salários mínimos. Em 2010, os índices de homicídio durante a adolescência foram de 2,19 por 100 mil habitantes 17.

A coorte foi acompanhada durante os 7-9 anos e 18-19 dos participantes. Utilizou-se dados do segundo segmento, realizado com os indivíduos aos seus 18-19 anos, em 2016. Todos os participantes incluídos na fase inicial do estudo, totalizando 2.443, foram captados em quatro juntas de alistamento militar na ilha de São Luís, no censo escolar de 2014 e em universidades.

Com o objetivo de aumentar o poder da amostra e prevenir perdas futuras, a coorte foi aberta para incluir outros indivíduos nascidos em São Luís, em 1997. A primeira etapa de busca ocorreu a partir de um sorteio, utilizando-se o banco de dados do Sistema de Informações sobre Nascidos Vivos (SINASC). Os critérios utilizados para o cadastro dos participantes foram: ter nascido em maternidade, na cidade de São Luís e em 1997. Com base nesta listagem, foi feito um sorteio aleatório, obtendose um total de 4.593 nascidos em 1997 na cidade de São Luís. Desse total, foi possível fazer contato telefônico ou pessoal com 1.13318 .

Em uma segunda etapa, 695 voluntários nascidos em 1997 foram identificados em escolas, universidades e mídias sociais, totalizando 1.828 adolescentes. Os membros do componente retrospectivo da coorte foram submetidos aos mesmos testes e questionários que os demais participantes da coorte original. Além disso, foi aplicado um questionário às mães destes adolescentes para coletar dados perinatais de forma retrospectiva. A amostra total foi de 2.515 participantes residentes em São Luís 18 .

Foram contratados e treinados profissionais da área da saúde para a aplicação dos questionários da pesquisa, por meio de entrevista face a face. Foram utilizadas questões referentes aos aspectos individuais, familiares e sociais dos adolescentes, bem como sobre a experiência destes com violência psicológica e eventos estressores. Ainda, abordou-se no questionário condições sociodemográficas, hábitos de vida, composição corporal, qualidade do sono, atividade física, habilidade cognitiva e transtornos mentais. Fizeram parte desta pesquisa 2.486 adolescentes.

A variável de exposição foi a violência psicológica sofrida nos 12 meses anteriores à apresentação de sintomas de TEPT. Para estimá-la, foi utilizada escala de violência psicológica 7 , que avalia experiências em que a vítima é desqualificada em suas capacidades, potencialidades, desejos e emoções e, ainda, cobrada excessivamente por uma pessoa significativa ao seu relacionamento. É constituída por 18 itens com opções de respostas que variam de "nunca" a "sempre" e foi adaptada transculturalmente à realidade brasileira, apresentando propriedades psicométricas de alfa de Cronbach de 0,91 e coeficiente de correlação intraclasse (ICC) de 0,8629 19. O escore da escala de violência psicológica foi definido como a razão entre o somatório dos pontos associados às frequências de cada um dos itens (1: nunca; 5: sempre) e a pontuação máxima que seria alcançada multiplicada por 100 . O ponto de corte foi determinado como valores acima e abaixo do terceiro quartil. Considerou-se a categoria de referência como violência não severa (raramente/às vezes) e severa (quase sempre/sempre).

A variável de desfecho foi o TEPT, que foi definido segundo o Manual Diagnóstico e Estatístico de Transtornos Mentais - 4a Edição (DSM-IV) da Associação Americana de Psiquiatria (APA) 20. Foi utilizado o questionário Mini (Entrevista Neuropsiquiátrica Internacional) autoaplicado. Trata-se de uma entrevista diagnóstica breve, padronizada e compatível com os critérios do DSM-IV e da Classificação Internacional de Doenças - 10a revisão (CID-10). Utilizou-se a versão plus do Mini, uma vez que ela é mais detalhada e compreende 19 módulos que exploram 17 transtornos do eixo I do DSM-IV 20. Além disso, ela aborda sistematicamente todos os critérios de inclusão e de exclusão e a cronologia (data do início e a duração dos transtornos, bem como o número de episódios) de 23 categorias diagnósticas do DSM-IV. No entanto, para esta pesquisa, foi utilizado apenas o módulo I (Transtorno de Estresse Pós-Traumático), que tem cinco itens de "sim" ou "não" e considera o início dos sintomas a partir dos 30 dias anteriores à pesquisa. Foram considerados para diagnóstico aqueles que responderam "sim" 
nos itens: características subjetivas de exposição ao trauma; revivência do evento traumático; esquiva persistente de estímulos associados ao evento e entorpecimento da responsividade geral; sintomas persistentes de excitabilidade.

Entre o conjunto de covariáveis utilizadas estão os fatores econômicos e sociodemográficos dos adolescentes, representados por: sexo; idade completa em anos; cor da pele (branca; não branca); índice de massa corporal - IMC (obesidade grau I: 30,0-34,9; obesidade grau II: 35,0-39,9; obesidade grau III: $\geq 40$ ); escolaridade (1 a 4; 5 a 8; 9 a 11 anos; 12 anos ou mais); classe econômica, seguindo o critério de classificação econômica da Associação Brasileira de Empresas de Pesquisa - ABEP (estratos $\mathrm{A}, \mathrm{B}$ e $\mathrm{C}$, correspondendo às camadas alta e média; $\mathrm{D} / \mathrm{E}$ às camadas populares); fatores estruturais da família (pais separados: sim ou não; chefe de família: pai, mãe ou outros; situação conjugal do adolescente: com ou sem companheiro; atividade laboral: sim ou não; prática religiosa: sim ou não; nem trabalha nem estuda: sim ou não); fatores comportamentais (uso de drogas ilícitas: sim ou não; consumo de álcool: < 8 baixo risco; e $\geq 8$ alto risco, utilizando o Alcohol Use Disorder Identification Test AUDIT) 21. Para identificar eventos estressores, foram considerados oito itens incluídos no questionário autoaplicado e que consistem em: adoecimento que resultou em afastamento das atividades diárias por mais de um mês; internação hospitalar devido à ocorrência de doença ou acidente; óbito de parente próximo; problemas financeiros severos; mudança forçada de moradia; separação ou divórcio; agressão física; assalto ou roubo. Os itens foram mensurados usando uma lista com respostas dicotômicas (sim ou não).

O plano de análise deste manuscrito foi iniciado com a análise dos fatores de confundimento ou de colisão e a seleção das variáveis de ajuste. Para tanto, utilizou-se o gráfico acíclico direcionado (DAG), por meio do software DAGitty 2.2 (http://www.dagitty.net/). O DAG codifica uma teoria ou pressupostos acerca da estrutura causal de um problema com base na literatura. Utilizá-lo na modelagem causal reforça a noção de que a causalidade implica direcionalidade de influência. Por meio de regras gráficas, utilizou-se o critério da porta de trás para identificar o conjunto mínimo de variáveis necessárias para o ajuste para viés de confundimento, ao mesmo tempo que se evitou o ajuste para colisores, a fim de não provocar viés de colisão 22 . As variáveis incluídas no conjunto de ajuste mínimo indicadas pelo DAG foram: sexo, classe econômica, situação conjugal dos pais, prática religiosa, evento estressor, consumo de alcool e uso de drogas ilícitas. A Figura 1 apresenta as variáveis incluídas no conjunto de ajuste mínimo indicadas pelo DAG.

As variáveis identificadas pelo DAG foram então utilizadas para a obtenção da homogeneidade entre os grupos de comparação - expostos e não expostos a violência psicológica - em relação às covariáveis. Para isso, foi utilizado o escore de propensão (EP) 23,24 em duas etapas. Inicialmente, o EP foi definido segundo as probabilidades condicionais de um indivíduo ser exposto, isto é, de sofrer violência psicológica, dado o conjunto de covariáveis observadas. Ele é sumarizado por uma única variável que considera simultaneamente todas as potenciais covariáveis de confusão, neste caso indicadas pelo DAG. Assim, indivíduos com o mesmo EP têm a mesma distribuição de covariáveis observadas, independente de terem sofrido ou não violência. O EP foi estimado por meio de regressão logística binária com o método de verossimilhança.

Em seguida, utilizou-se o EP para a criação do inverso da probabilidade de seleção (IPS). Dessa forma, atribuiu-se uma probabilidade para cada adolescente, sendo 1/IPS para aqueles que compõem o grupo de expostos e 1/(1-IPS) para aqueles do grupo dos não expostos 23,24. Essa condição tornou os dois grupos mais homogêneos e comparáveis entre si.

Para os dois grupos foi estimada a proporção das covariáveis selecionadas para compor o modelo de estimação do EP antes e depois da ponderação pelo IPS, a fim de verificar o padrão de distribuição dessas covariáveis entre os grupos de estudo. Análises com o teste qui-quadrado de Pearson foram realizadas para verificar o nível de significância estatística do desbalanceamento das covariáveis antes e depois do controle pelo EP, alcançando-se homogeneidade quando a probabilidade do teste foi > 0,05

Para verificar a associação entre as variáveis de confundimento e a variável de exposição (violência psicológica), foi utilizado modelo de regressão logística múltiplo - nível de significância de 5\%. As prevalências e os seus intervalos de 95\% de confiança (IC95\%) foram estimadas para a ocorrência de TEPT segundo a variável de exposição, tanto antes quanto depois da ponderação pelo IPS. A associação entre a ocorrência de violência psicológica e TEPT foi estimada pela odds ratio (OR), pelo IC95\% e por meio da regressão logística binária bruta e ajustada, ponderada pelo IPS. No método de escore 


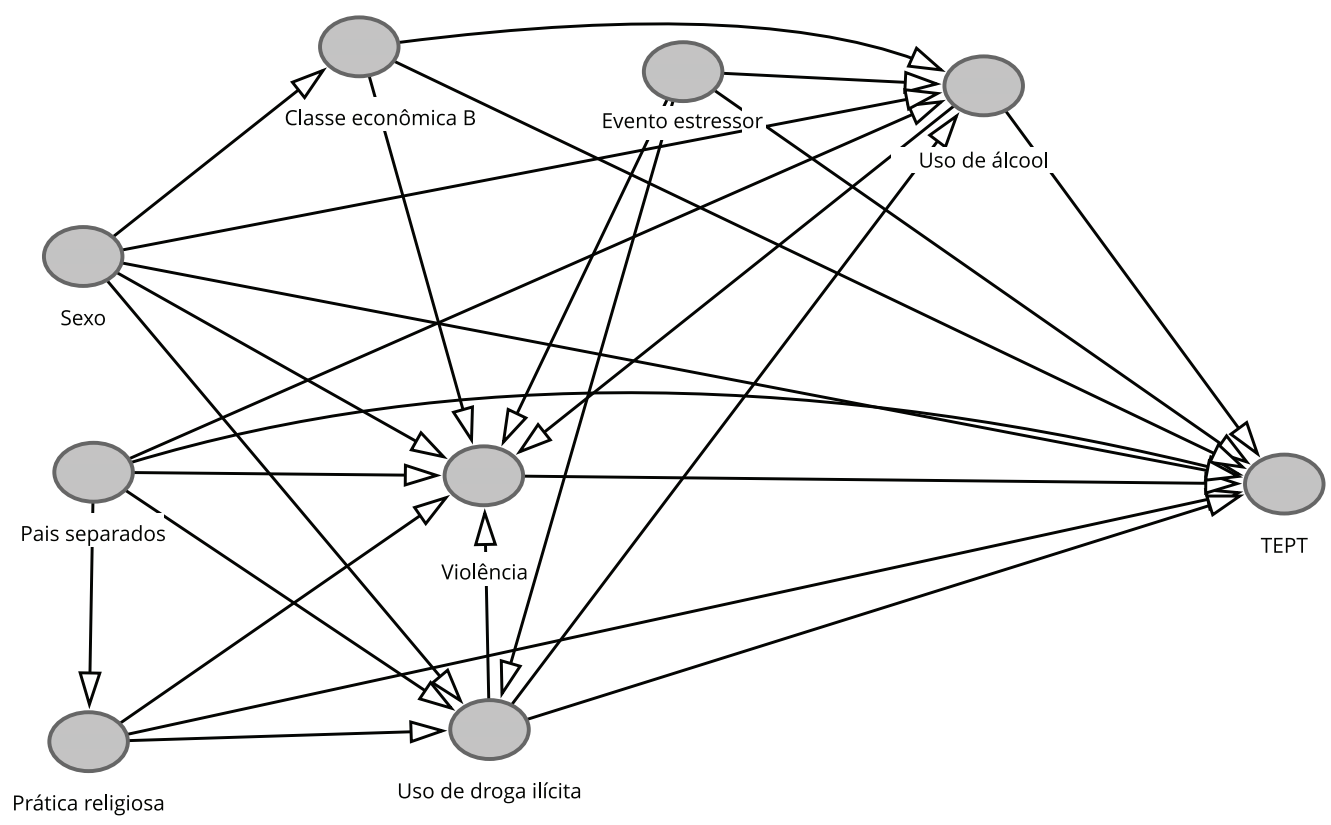

TEPT: transtorno de estresse pós-traumático.

de propensão implementado, inclusive com ponderação (IPS), considerou-se o efeito médio do tratamento (ATE; average treatment effects) como o tipo de estimador de inferência causal. As análises foram realizadas no software Stata, versão 14.0 (https://www.stata.com).

O segundo segmento atendeu aos critérios da Resolução no 466/2012 do Conselho Nacional de Saúde e os adolescentes que concordaram em participar da pesquisa assinaram o Termo de Consentimento Livre e Esclarecido (TCLE). O projeto e o TCLE foram aprovados pelo Comitê de Ética em Pesquisa do Hospital Universitário da UFMA (parecer consubstanciado no 1.302 .489 de 29 de outubro de 2015).

\section{Resultados}

Entre os 2.486 adolescentes estudados, 30,3\% experienciaram violência psicológica na forma severa. Ademais, 2,5\% atestaram TEPT, sendo que 4,8\% estão entre os expostos à violência psicológica e 1,5\% entre os não expostos. Essa diferença foi estatisticamente significativa ( $\mathrm{p}<0,001)$, como mostra a Tabela 1. Foram excluídos 318 adolescentes com dados faltantes (missings) em algumas das variáveis estudadas na análise.

Os fatores associados à violência psicológica severa, segundo a análise de regressão logística múltipla, foram: sexo feminino (OR = 1,52; IC95\%: 1,25-1,85), consumo de álcool (OR = 1,82; IC95\%: 1,42-2,33), uso de drogas ilícitas ( $\mathrm{OR}=1,66$; IC95\%: 1,33-2,08) e a presença de evento estressor, fator que mais se associou à ocorrência de violência psicológica severa (OR = 2,77; IC95\%: 2,25-3,40).

Na Tabela 2, apresentam-se as covariáveis utilizadas no ajuste antes e depois de ponderar pelo IPS. Por meio do teste qui-quadrado de Pearson, observou-se o nível de significância estatística do desbalanceamento das covariáveis antes do controle pelo EP e a recuperação da homogeneidade da distribuição destas covariáveis entre os grupos. A homogeneidade foi observada pela probabilidade 
Tabela 1

Distribuição da violência psicológica e do transtorno de estresse pós-traumático (TEPT) em adolescentes ( $N=2.486$ ). São Luís, Maranhão, Brasil, 2018.

\begin{tabular}{|c|c|c|c|c|c|c|c|}
\hline \multirow[t]{3}{*}{ Violência psicológica severa } & \multicolumn{6}{|c|}{ TEPT } & \multirow[t]{3}{*}{ Valor de $p$ * } \\
\hline & \multicolumn{2}{|c|}{ Sim } & \multicolumn{2}{|c|}{ Não } & \multicolumn{2}{|c|}{ Total } & \\
\hline & $\mathbf{n}$ & $\%$ & $\mathbf{n}$ & $\%$ & $\mathbf{n}$ & $\%$ & \\
\hline Sim & 37 & 4,8 & 735 & 95,2 & 772 & 30,3 & 0,0001 \\
\hline Não & 25 & 1,5 & 1.689 & 98,5 & 1.714 & 69,7 & \\
\hline Total & 62 & 2,5 & 2.424 & 97,5 & 2.486 & 100,0 & \\
\hline
\end{tabular}

* Teste qui-quadrado de Pearson.

\section{Tabela 2}

Características de adolescentes ( $N=2.486$ ) que sofreram ou não violência psicológica severa antes e depois de ponderar pelo inverso da probabilidade de seleção (IPS) do escore de propensão estimado. São Luís, Maranhão, Brasil, 2018.

\begin{tabular}{|c|c|c|c|c|c|c|}
\hline \multirow[t]{3}{*}{ Covariáveis } & \multicolumn{3}{|c|}{ Amostra antes da ponderação pelo IPS } & \multicolumn{3}{|c|}{ Amostra depois da ponderação pelo IPS } \\
\hline & \multicolumn{2}{|c|}{$\begin{array}{c}\text { Exposição à violência psicológica } \\
\text { severa (\%) }\end{array}$} & \multirow[t]{2}{*}{ Valor de $p$} & \multicolumn{2}{|c|}{$\begin{array}{c}\text { Exposição à violência psicológica } \\
\text { severa (\%) }\end{array}$} & \multirow[t]{2}{*}{ Valor de $\mathrm{p}$} \\
\hline & $\operatorname{Sim}(n=772)$ & Não $(n=1.720)$ & & $\operatorname{Sim}(n=1.827)$ & Não (n = 1.507) & \\
\hline \multicolumn{7}{|l|}{ Sexo } \\
\hline Feminino & 34,2 & 65,8 & \multirow{2}{*}{0,001} & 49,5 & 50,5 & \multirow{2}{*}{0,72} \\
\hline Masculino & 27,6 & 72,4 & & 50,3 & 49,7 & \\
\hline \multicolumn{7}{|l|}{ Classe econômica } \\
\hline$A$ & 34,4 & 6,56 & \multirow{4}{*}{0,641} & 56,6 & 43,4 & \multirow{4}{*}{0,42} \\
\hline B & 29,3 & 70,7 & & 47,3 & 52,7 & \\
\hline C & 31,9 & 68,1 & & 50,9 & 49,1 & \\
\hline $\mathrm{D} / \mathrm{E}$ & 31,9 & 68,1 & & 49,1 & 50,9 & \\
\hline \multicolumn{7}{|c|}{ Situação conjugal dos pais } \\
\hline Sem companheiro & 33,7 & 66,3 & \multirow{2}{*}{0,006} & 49,9 & 50,1 & \multirow{2}{*}{0,99} \\
\hline Com companheiro & 28,5 & 71,5 & & 49,9 & 50,1 & \\
\hline \multicolumn{7}{|l|}{ Prática religiosa } \\
\hline Não & 34,3 & 65,7 & \multirow{2}{*}{0,023} & 49,8 & 50,2 & \multirow{2}{*}{0,95} \\
\hline Sim & 29,7 & 70,3 & & 49,9 & 50,1 & \\
\hline \multicolumn{7}{|l|}{ Evento estressor } \\
\hline Não & 24,6 & 75,4 & \multirow{2}{*}{0,001} & 49,9 & 50,1 & \multirow{2}{*}{0,99} \\
\hline Sim & 49,5 & 50,5 & & 49,9 & 50,1 & \\
\hline \multicolumn{7}{|l|}{ Consumo de álcool } \\
\hline Baixo risco $(<8)$ & 27,4 & 72,6 & \multirow{2}{*}{0,001} & 49,9 & 50,1 & \multirow{2}{*}{0,95} \\
\hline Alto risco $(\geq 8)$ & 46,0 & 54,0 & & 49,9 & 50,1 & \\
\hline \multicolumn{7}{|l|}{ Uso de drogas ilícitas } \\
\hline Não & 26,7 & 73,3 & \multirow{2}{*}{0,001} & 49,8 & 50,2 & \multirow{2}{*}{0,90} \\
\hline Sim & 43,6 & 56,4 & & 50,1 & 49,9 & \\
\hline
\end{tabular}


do teste, que foi > 0,05 para todas as covariáveis após o controle pelo EP. As covariáveis utilizadas no ajuste foram: sexo feminino, ter pais separados, não ter práticas religiosas, ter hábitos de vida não saudáveis (consumo de álcool e de drogas ilícitas) e ter presenciado eventos estressores, com a exceção da classe econômica.

Por fim, a Tabela 3 demonstra a estimação do impacto da violência psicológica na forma severa sobre o TEPT. Observou-se uma associação da violência psicológica severa com o TEPT nas duas análises realizadas, porém a magnitude no modelo estruturado pelo EP ( $\mathrm{OR}=1,97$; IC95\%: 1,08-3,56) indicou um ajuste da medida de associação da análise bruta ( $\mathrm{OR}=3,40$; IC95\%: 2,03-5,69). Adolescentes vítimas de violência psicológica severa tiveram 1,97 mais chances de desenvolver o TEPT após a regressão binária estruturada ponderada pelo IPS.

\section{Discussão}

Este estudo analisou o risco de desenvolvimento de TEPT associado à ocorrência de violência psicológica severa. A violência psicológica do tipo severa esteve associada significativamente ao risco aumentado de desenvolvimento do TEPT. Fatores como ser do sexo feminino, ter hábitos de vida não saudáveis, como o consumo de álcool e de drogas ilícitas, e ter sofrido algum evento estressor estiveram associados à ocorrência de violência psicológica severa. Nesse sentido, este estudo contribui para a escassa literatura sobre a exposição de violência psicológica e a sua associação com o desenvolvimento do TEPT, confirmando o impacto negativo dessa forma de abuso na saúde mental do indivíduo.

A associação estreita entre o TEPT e a violência física e sexual é amplamente citada na literatura 2,5,8,14. Contudo, a violência psicológica se torna mais prejudicial devido ao seu caráter silencioso e pelo fato de, muitas vezes, a vítima não ter evidências físicas constatáveis, o que torna difícil a avaliação 8,14. Adolescentes do sexo feminino que testemunharam ou sofreram algum tipo de violência podem ser acometidas por um envolvimento emocional maior em comparação aos adolescentes do sexo masculino. Assim, a influência da resposta ao estresse fisiológico na mulher pode ser mais exacerbada, o que pode gerar uma resposta bem mais intensa $1,6,25,26$.

Registros na literatura mostram que indivíduos que fazem uso abusivo de álcool, sobretudo mulheres vítimas de violência sexual e física, estão muito mais propensos a sofrer de TEPT do que a população em geral 27,28,29. Os problemas de comportamento podem ser tanto os preditores para a exposição à violência quanto as consequências desta exposição. Nestes casos, forma-se uma relação bidirecional 30,31. Devido ao seu componente emocional, aquelas que consomem álcool de forma abusiva geralmente apresentam comportamentos impulsivos que as tornam vulneráveis a maus tratos, principalmente entre os seus responsáveis, o que fragiliza ainda mais as relações familiares 32,33.

Situações como esta comprometem a autoconfiança que o adolescente desenvolve quando é apoiado emocionalmente em suas relações sociais, religiosas e, principalmente, em seu ambiente familiar. Essas relações de apoio permitem que o adolescente lide melhor com as experiências trau-

\section{Tabela 3}

Transtorno de estresse pós-traumático (TEPT) associado à ocorrência de violência psicológica severa segundo a regressão logística bruta e estruturada pelo inverso da probabilidade de seleção (IPS) do escore de propensão estimado de adolescentes ( $N$ = 2.486). São Luís, Maranhão, Brasil, 2016.

\begin{tabular}{lcccc}
\hline Exposição à violência psicológica severa & \multicolumn{2}{c}{ TEPT } \\
& \multicolumn{2}{c}{ Análise bruta } & \multicolumn{2}{c}{ Ponderada pelo IPS } \\
& OR & IC95\% & OR & IC95\% \\
\hline Não & - & - & - & - \\
Sim & 3,40 & $2,03-5,69$ & 1,97 & $1,08-3,56$ \\
\hline
\end{tabular}

IC95\%: intervalo de 95\% de confiança; OR: odds ratio. 
máticas e com o estresse advindo delas. É certo que o desenvolvimento da resiliência individual está diretamente relacionado com a capacidade de lidar com os eventos e controlar os seus resultados 2,5,32,33. Estudos comprovaram que situações que promovem a capacidade de se adaptar a diferentes tipos de eventos estressores funcionam como fatores protetores nesse contexto 27,28,29.

Sobre os eventos estressores associados à violência psicológica, a nossa análise destacou que a maioria referiu a insegurança e o consequente medo do entorno da área habitacional em casos de assaltos com o uso de armas de fogo ou da morte de uma pessoa próxima. Em sua maior parte, esses preditores geram alterações na saúde mental e comportamental, desenvolvendo-se sob forma de ansiedade, depressão e dependência de álcool e drogas, além de abandono escolar e dificuldades recorrentes de estabelecer relações interpessoais, enfatizando a incessante busca de autoafirmação 16,33,34.

Nesse ambiente adverso, o adolescente se vê sem qualquer expectativa real ou aparente de futuro 34,35. Como consequência imediata, o vitimado assume uma postura quase letárgica, o que compromete os seus sentimentos de realização tanto educacional quanto ocupacional e até mesmo a sua felicidade em viver 16,25,35. Experiências comprovam, ainda, o desenvolvimento de estados depressivos entre as vítimas de violência tanto física quanto psicológica, sendo esta a menos detectável e a mais ignorada 36,37. Tais efeitos são reforçados em estudos sobre desempenho educacional ao se referirem às expressões traumáticas e psicológicas advindas da experiência violenta 38,39,40.

Zhi et al. 25, ao se aprofundarem em um universo composto de 4.215 adolescentes na faixa etária de 12 a 20 anos expostos à violência psicológica, identificaram a formação de um estado permanente de violência que geralmente encontra a sua resposta nas agressões verbais e nos ataques interpessoais, comportamento comum entre os grupos sociais constituídos por essas gerações. Acompanhando a mesma linha de raciocínio, Han et al. 41 acrescentam o baixo desempenho escolar e a aquisição de maus hábitos, como o consumo de drogas.

Estudos realizados sobre os sintomas do estresse pós-traumático expuseram as mesmas consequências destruidoras e permanentes 9,42,43. Jovens adolescentes oriundos de ambientes adversos e expostos a violências físicas e/ou psicológicas invariavelmente desenvolvem sentimentos negativos e confusos sobre o futuro, o que os tornam mais propensos a agressões, bem como a praticarem atos violentos iguais aos vivenciados na vida tanto doméstica quanto comunitária 10,38,41.

Este estudo utilizou o modelo de EP como estratégia metodológica, uma vez que é utilizado em estudos de inferência causal, o que possibilitou a homogeneidade dos grupos da amostra, a permutabilidade adequada entre eles e o controle dos possíveis fatores de confundimento. Observou-se uma associação da violência psicológica severa com o TEPT nas duas análises realizadas, porém a magnitude no modelo estruturado pelo EP indicou um ajuste da medida de associação da análise bruta, confirmando com maior robustez tal associação. O tempo de exposição, no caso, à violência psicológica, precedeu em 12 meses os sintomas de TEPT, o que nos permitiu traçar a linha da temporariedade.

Como outro ponto forte, o tamanho da amostra é representativo e de base populacional, proporcionando correlações significativas. Este estudo abordou apenas a população adolescente em uma fase mais tardia da adolescência, cuja avaliação cognitiva pode ser mais bem percebida. Assim, foram utilizados instrumentos específicos, adaptados à nossa realidade sociocultural. A utilização do DAG para a construção do modelo teórico e de seleção do conjunto mínimo de covariáveis necessárias para o ajuste compôs o conjunto de técnicas analíticas utilizadas.

Como limitação deste estudo, podemos citar que foram utilizados para diagnóstico de TEPT os critérios centrais do TEPT, conforme delineados pelo DSM-IV 20. Mais recentemente, o diagnóstico de TEPT com o DSM-5 44 sofreu mudanças importantes. Porém, as alterações dizem respeito, especialmente, às crianças pré-escolares 44. De acordo com os critérios do DSM-IV, a ocorrência do TEPT em crianças em idade pré-escolar pode ser subestimada em relação às crianças mais velhas e adultos, possivelmente devido às percepções imaturas das crianças aos eventos ou à baixa sensibilidade dos critérios atuais para detectar as peculiaridades das manifestações do TEPT 45.

Em um estudo comparativo utilizando uma amostra populacional, os autores observaram que, apesar das evidências mostrarem ser consistentes com o diagnóstico de DSM-5, não houve diferença substancial em termos de prevalência com base nos critérios do DSM-IV 46. Os adolescentes, em nossa amostra, foram investigados praticamente ao final de sua adolescência, o que contribuiu para que eles tivessem melhor discernimento em relação à percepção dos sintomas e aos eventos desencadeadores de TEPT. 


\section{Considerações finais}

Adolescentes vítimas de violência psicológica têm um risco substancial de desenvolver TEPT. Deve-se levar em conta as mudanças no desenvolvimento sociocultural e as diferentes medidas contextuais com relação às medidas de avaliação. Dada a escassez de avaliações sobre essa diferente forma de violência e a sua significativa associação ao TEPT, este estudo buscou enfatizar a importância da identificação desses adolescentes apontando metas específicas de tratamento. A contribuição principal dessa abordagem é a identificação das exposições de maneira precoce, proporcionando às vítimas a capacidade de gerenciamento sobre as diferentes respostas que venham a apresentar. Isso pode garantir o controle das manifestações tanto no âmbito cognitivo quanto somático de sofrimento, bem como o possível controle das consequências desse tipo de transtorno, sobretudo na vida adulta.

\section{Colaboradores}

L. G. G. S. Álvares participou do planejamento e desenho do estudo, análise dos dados, escrita e revisão do manuscrito. M. T. S. S. B. Alves participou do planejamento e desenho do estudo, análise dos dados e revisão do manuscrito. A. M. Santos participou do planejamento do estudo, análise dos dados e revisão do manuscrito. B. L. C. A. Oliveira e D. C. Chagas participaram da análise dos dados e revisão do manuscrito. Todos os autores aprovaram a versão final do manuscrito.

\section{Informações adicionais}

ORCID: Livia Goreth Galvão Serejo Álvares (00000002-0884-190X); Maria Teresa Seabra Soares de Britto e Alves (0000-0002-4806-7752); Alcione Miranda dos Santos (0000-0001-9711-0182); Bruno Luciano Carneiro Alves de Oliveira (0000-00018053-7972); Deysianne Costa das Chagas (00000003-0239-6662).

\section{Agradecimentos}

Ao Departamento de Ciência e Tecnologia do Ministério da Saúde (DECIT/MS), ao Conselho Nacional de Desenvolvimento Científico e Tecnológico $(\mathrm{CNPq})$ e à Fundação de Amparo à Pesquisa e ao Desenvolvimento Científico e Tecnológico do Maranhão (FAPEMA) pelo financiamento e ao Hospital Universitário da Universidade Federal do Maranhão pelo apoio na logística da coleta de dados. 


\section{Referências}

1. Mclaughlin KA, Koenen KC, Hill ED, Petukhova M, Sampson NA, Zaslavsky AM, et al. Trauma exposure and posttraumatic stress disorder in a national sample of adolescents. J Am Acad Child Adolesc Psychiatry 2013; 52:815-30.

2. Miller-Graff LE, Scrafford K, Rice C. Conditional and indirect effects of age of first exposure on PTSD symptoms. Child Abuse Negl 2016; 51:303-12.

3. Kearney CA, Wechsler A, Kaur H, LemosMiller A. Posttraumatic stress disorder in maltreated youth: a review of contemporary research and thought. Clin Child Fam Psychol Rev 2010; 13:46-76.

4. Carrion VG, Kletter H. Post-traumatic stress disorder: shifting toward a developmental framework. Child Adolesc Psychiatric Clin N Am 2012; 21:573-91.

5. Abranches CD, Assis SG. A (in)visibilidade da violência psicológica na infância e adolescência no contexto familiar. Cad Saúde Pública 2011; 27:843-54.

6. Satyanarayana VA, Prabha SC, Krishna V. Mental health consequences of violence against women and girls. Curr Opin Psychiatry 2015; 28:350-6.

7. Pitzner JK, Drummond PD. The reliability and validity of empirically scaled measures of psychological/verbal control and physical/sexual abuse: relationship between current negative mood and a history of abuse independent of other negative life events. J Psychosom Res 1997; 43:125-42.

8. Panuzio J, Taft CT, Black DA, Murphy CM, Koenen KC. Relationship abuse and victims posttraumatic stress disorder symptoms: associations with child behavior problems. J Fam Viol 2007; 22:177-85.

9. Bordin IA, Duarte CS, Ribeiro WS, Paula CS, Coutinho ESF, Sourander A, et al. Violence and child mental health in Brazil: the itaboraí youth study methods and findings. Int J Methods Psychiatr Res 2018; 27:e1605.

10. Teche SP, Barros AJ, Rosa RG, Guimarães LP, Cordini KL, Goi JD, et al. Association between resilience and posttraumatic stress disorder among Brazilian victims of urban violence: a cross-sectional case-control study. Trends Psychiatry Psychother 2017; 39:116-23.

11. Luz MP, Coutinho ES, Berger W, Mendlowicz MV, Vilete LM, Melloet MF, et al. Conditional risk for posttraumatic stress disorder in an epidemiological study of a Brazilian urban population. J Psychiatr Res 2016; 72:51-7.

12. Pupo MC, Serafim PM, Mello MF. Healthrelated quality of life in posttraumatic stress disorder: 4 years follow-up study of individuals exposed to urban violence. Psychiatry Res 2015; 228:741-5.

13. Lewis SJ, Arseneault L, Caspi A, Fisher HL, Matthews T, Moffitt TE, et al. The epidemiology of trauma and post-traumatic stress disorder in a representative cohort of young people in England and Wales. Lancet Psychiatry 2019; 6:247-56.
14. Sullivan TP, Fehon DC, Andres-Hyman RC, Lipschitz DS, Grilo CM. Differential relationships of childhood abuse and neglect subtypes to PTSD symptom clusters among adolescent inpatients. J Trauma Stress 2002; 19:229-39.

15. Ximenes LF, Oliveira RV, Assis SG. Violência e transtorno de estresse pós-traumático na infância. Ciênc Saúde Colet 2009; 14:417-33.

16. Avanci JQ, Serpeloni F, Oliveira TP, Assis SG. Posttraumatic stress disorder among adolescents in Brazil: a cross-sectional study. BMC Psychiatry 2021;21:75.

17. Instituto Brasileiro de Geografia e Estatística. Estimativas da população dos municípios brasileiros com data de referência em 1o de julho de 2014. https://www.ibge.gov.br/arquivo/ noticias/pdf/analise_ estimativas_2014.pdf (acessado em 14/Jun/2018).

18. Simões VMF, Batista RFL, Alves MTSSB, Ribeiro CCC, Thomaz EBAF, Carvalho CA, et al. Saúde dos adolescentes da coorte de nascimentos de São Luís, Maranhão, Brasil, 1997/1998. Cad Saúde Pública 2020; 36:e00164519.

19. Pesce RP, Assis SG, Avanci JQ, Santos NC, Malaquias JV, Carvalhaes R. Adaptação transcultural, confiabilidade e validade da escala de resiliência. Cad Saúde Pública 2005; 21:436-48.

20. Associação Americana de Psiquiatria. Manual diagnóstico e estatístico de transtornos mentais. 4a Ed. Porto Alegre: Artmed; 2003.

21. Babor TF, Higgins-Biddle JC, Saunders JB, Monteiro MG. The alcohol use disorders identification test: guideline for use in primary care. 2nd Ed. Geneva: World Health Organization; 2001.

22. Fleischer NL, Diez Roux AV. Using directed acyclic graphs to guide analyses of neighbourhood health effects: an introduction. J Epidemiol Community Health 2008; 62:842-6.

23. Oakes JM, Johnson P. Propensity score matching for social epidemiology. In: Oakes JM, Johnson P, editors. Methods in social epidemiology. San Francisco: Jossey-Bass; 2006. p. 370-92.

24. Hahs-Vaughn DL. Propensity score analysis with complex survey samples. In: Pan W, Bai $\mathrm{H}$, editors. Propensity score analysis: fundamentals and developments. New York: Guilford Press; 2015. p. 237-64.

25. Zhi K, Yang J, Chen Y, Akebaijiang N, Liu M, Yang X, Zhang S. The relationship between future time perspective and psychological violence among chinese college students. Front Psychol 2021; 12:585837.

26. Xia Y, Li SD, Liu T. The interrelationship between family violence, adolescent violence, and adolescent violent victimization: an application and extension of the cultural spillover theory in China. Int J Environ Res Public Health 2018; 15:371.

27. Carbone JT, Holzer KJ, Vaughn MG. Posttraumatic stress disorder among low-income adolescents experiencing family-neighborhood income disparities. J Trauma Stress 2019; 32:899-907. 
28. Petrakis IL, Simpson TL. Posttraumatic stress disorder and alcohol use disorder: a critical review of pharmacologic treatments. Alcohol Clin Exp Res 2017; 41:226-37.

29. Goldstein B, Bradley B, Ressler KJ, Powers A. Associations between posttraumatic stress disorder, emotion dysregulation, and alcohol dependence symptoms among inner city females. J Clin Psychol 2017; 73:319-30.

30. Trichett PK, Mennen FE, Kim K, Sang J. Emotional abuse in a sample of multiply maltreated, urban young adolescents: issues of definition and identification. Child Abuse Negl 2009; 33:27-35.

31. Mikton CR, Butchart A, Dahlberg LL, Krug EG. Global status report on violence prevention 2014. Am J Prev Med 2016; 50:652-9.

32. Carlson EB, Palmieri PA, Field NP, Dalenberg CJ, Macia KS, Spain DA. Contributions of risk and protective factors to prediction of psychological symptoms after traumatic experiences. Compr Psychiatry 2016; 69:106-15.

33. Smith P, Dalgleish T, Meiser-Stedman R. Practitioner review: posttraumatic stress disorder and its treatment in children and adolescents. J Child Psychol Psychiatry 2019; 60:500-15.

34. Lee DB, Hsieh HF, Stoddard SA, Heinze JE, Carter PM, Goldstick JE, et al. Longitudinal pathway from violence exposure to firearm carriage among adolescents: the role of future expectation. J Adolesc 2020; 81:101-13.

35. Cloitre M, Hyland P, Bisson JI, Brewin CR, Roberts NP, Karatzias T, et al. ICD-11 posttraumatic stress disorder and complex posttraumatic stress disorder in the United States: a population-based study. J Trauma Stress 2019; 32:833-42.

36. Ross J, Armour C, Kerig PK, Kidwell MC, Kilshaw RE. A network analysis of posttraumatic stress disorder and dissociation in trauma-exposed adolescents. J Anxiety Disord 2020; 72:102222.

37. Koen N, Brittain K, Donald KA, Barnett W, Koopowitz S, Maré K, et al. Psychological trauma and posttraumatic stress disorder: risk factors and associations with birth outcomes in the Drakenstein Child Health Study. Eur J Psychotraumatol 2016; 7:28720.
38. Zerach G, Elklit A. Polyvictimization and psychological distress in early adolesccence: a mediation model of defense mechanisms and coping styles. J Interpers Violence 2017; 35:4732-56.

39. Charak R, de Jong JTVM, Berckmoes LH, Ndayisaba H, Reis R. Assessing the fator structure of the Childhood Trauma Questionnaire, and cumulative effect of abuse and neglect on mental health among adolescents in conflictaffected Burundi. Child Abuse Negl 2017; 72:383-92.

40. Guerra C, Pereda N, Guilera G, Abad J. Internalizing symptoms and polyvictimization in a clinical sample of adolescents: the roles of social support and non-productive coping strategies. Child Abuse Negl 2016; 54:57-65.

41. Han Z, Zhang G, Zhang H. School bullying in urban China: prevalence and correlation with school climate. Int J Environ Res Public Health 2017; 14:1116.

42. Greene CA, Chan G, McCarthy KJ, Wakschlag LS, Briggs-Gowan MJ. Psychological and physical intimate partner violence and young children's mental health: the role of maternal posttraumatic stress symptoms and parenting behaviors. Child Abuse Negl 2018; 77:168-79.

43. Ribeiro FML, Minayo MCS. O papel da religião na promoção da saúde, na prevenção da violência e na reabilitação de pessoas envolvidas com a criminalidade: revisão de literatura. Ciênc Saúde Colet 2014; 9:1773-89.

44. American Psychiatric Association. Diagnostic and statistical manual of mental disorders DSM-5. Washingnton DC: American Psychiatric Association; 2013.

45. Araújo AC, Lotufo Neto F. A nova classificação americana para os transtornos mentais: o DSM-5. Rev Bras Ter Comport Cogn 2014; 16:67-82.

46. Kilpatrick DG, Resnick HS, Milanak ME, Miller MW, Keyes KM, Friedman MJ. National estimates of exposure to traumatic events and PTSD prevalence using DSM-IV and DSM-5 criteria. J Trauma Stress 2013; 26:537-47. 


\section{Abstract}

The study analyzed the impact of psychological violence on the development of posttraumatic stress disorder (PTSD). This was a cross-sectional study nested in a cohort in which the second follow-up was conducted in 2016. A questionnaire was applied to 2,486 adolescents and approached individual, family, and social aspects and experience with psychological violence. Propensity score was used to create inverse probability weighting (IPW). Thus, a probability was assigned to each adolescent, where $1 / I P W$ represent those in the exposed group and 1/(1-IPW) those in the unexposed group. This procedure made the two groups more homogeneous and mutually comparable. The association between the occurrence of psychological violence and PTSD was estimated by odds ratios $(\mathrm{OR})$ and $95 \%$ confidence intervals (95\% CI) via crude binary logistic regression and adjusted logistic regression (weighted by IPW). According to the results, $30.3 \%$ reported having suffered severe violence. Prevalence of PTSD was $4.8 \%$ among exposed and $1.5 \%$ in unexposed to psychological violence. An association was observed between severe violence and PTSD in the two analyses, but the magnitude in the model structured by the propensity score (OR $=1.97$; 95\%CI: 1.08-3.56) indicated an adjustment to the measure from the crude analysis $(O R=3.40 ; 95 \% C I: 2.03-5.69)$. The current study contributes to the scarce literature on exposure to psychological violence and its association with the development of PTSD, confirming the negative impact of this form of abuse on the individual's mental health.

Adolescent; Violence; Post-Traumatic Stress Disorders

\section{Resumen}

Se analizó la influencia de la ocurrencia de violencia psicológica en el desarrollo de trastorno de estrés postraumático (TEPT). Se trató de un estudio transversal anidado en una cohorte, cuyo segundo seguimiento se realizó en 2016. Se aplicó un cuestionario en 2.486 adolescentes, a través del cual se abordaron aspectos individuales, familiares, sociales, así como de vivencia de violencia psicológica. Se utilizó el marcador de propensión para que se creara el inverso de la probabilidad de selección (IPS). De esta forma, se atribuyó una probabilidad para cada adolescente, siendo 1/IPS para aquellos que componen el grupo de expuestos y 1/(1-IPS) para aquellos del grupo no expuestos. Esta condición hizo los dos grupos más homogéneos y comparables entre sí. La asociación entre ocurrencia de violencia psicológica y TEPT se estimó mediante OR (odds ratio) y el IC95\% (intervalo de 95\% de confianza) por medio de la regresión logística binaria bruta y ajustada (ponderada por el IPS). Se verificó que un 30,3\% informaron haber sufrido violencia severa. La prevalencia del TEPT fue de un 4,8\% entre los expuestos contra un 1,5\% en no expuestos a la violencia psicológica. Se observó una asociación de la violencia severa con el TEPT en los dos análisis realizados, no obstante, la magnitud en el modelo estructurado por el marcador de propensión $(\mathrm{OR}=1,97$; IC95\%: 1,08-3,56) indicó un ajuste de la medida de asociación del análisis bruto $(O R=3,40$; IC95\%: 2,03-5,69). Este estudio contribuye a la escasa literatura sobre la exposición de violencia psicológica y su asociación con el desarrollo del TEPT, confirmando el impacto negativo de esa forma de abuso en la salud mental del individuo.

Adolescente; Violência; Trastornos por Estrés Postraumático
Recebido em 05/Out/2020

Versão final reapresentada em 21/Jun/2021 Aprovado em 08/Jul/2021 